COMMUNICATIONS IN

ANALYSIS AND GEOMETRY

Volume 8, Number 3, 531-543, 2000

\title{
Integral curvature bounds and bounded diameter
}

\section{Chadwick Sprouse}

We prove an analogue of Myers' diameter bound for Riemannian manifolds in the case where the Ricci curvature below a positive constant is small in an averaged sense. This improves several previous results for manifolds with small amounts of nonpositive curvature.

\section{Introduction.}

One of the earliest and most fundamental theorems relating local geometry to global geometry/topology is that of Myers [10], which states that a complete Riemannian manifold with Ricci curvature bounded from below by $(n-1) k>0$ is compact, with diameter $\leq \pi / \sqrt{k}$, and finite fundamental group. There have been several attempts to generalize this in various directions, the closest in spirit to the following being those of Elworthy-Rosenberg [5],[6], Rosenberg-Yang [14], and Wu [15] which extend the theorem to manifolds with small "wells of negative curvature". In these cases, the wells are assumed to be of either small diameter ([5], [15]), or small volume ([6], [14]). (Note that with this type of theorem it is also necessary to impose a restriction on the "depth" of the wells, such as a fixed but arbitrary lower Ricci curvature bound, as can be seen by attaching a very small handle to a sphere, or by attaching two spheres by a very small neck.) In addition, in [14] there are related generalizations of Bochner's vanishing theorem and Myers' $\pi_{1}$-finiteness result for wells bounded in $L^{1}$-norm.

Here, we show using an inequality of Cheeger-Colding that in fact for a complete Riemannian manifold with (nonpositive) lower Ricci curvature bounds, one has bounded diameter and finite fundamental group provided that the Ricci curvature below some positive constant is small in a suitable integral sense. This generalizes several of the results in the above papers. In particular, all theorems on the finiteness of $\pi_{1}(M)$ in the above are consequences of Theorem 1.3 below. Furthermore, [14, Theorem 11.1] and [15, Theorem 1] should be compared with Corollary 3.2 and Theorem 1.2, where we are able to bound the diameter of $M$ by a value arbitrarily close to $\pi$ without restricting the set of points on which the Ricci curvature can be nonpositive. 
For many recent results on manifolds with integral curvature bounds, see [11], [12], [13] and the references therein.

Notation: Let $\mathrm{Ric}_{-}(x)$ denote the lowest eigenvalue of the Ricci tensor, $\operatorname{Ric}_{x}$. $\left(M^{n}, g\right)$ will always be a complete $n$-dimensional Riemannian manifold. For an arbitrary function $f$ on $M, f_{+}(x)=\max \{f(x), 0\}$.

Theorem 1.1. Let $(M, g)$ be a compact Riemannian manifold with Ric $\geq 0$. Then for any $\delta>0$ there exists $\varepsilon=\varepsilon(n, \delta)$ such that if

$$
\frac{1}{\operatorname{vol}(M)} \int_{M}\left((n-1)-\mathrm{Ric}_{-}\right)_{+} d V<\varepsilon(n, \delta),
$$

then $\operatorname{diam}(M)<\pi+\delta$.

In the case that $(M, g)$ is noncompact or does not possess nonnegative Ricci curvature, one can achieve a similar result by averaging the 'bad' part of Ric over metric balls.

Theorem 1.2. Let $(M, g)$ be a complete Riemannian manifold with Ric $\geq$ $(n-1) k(k \leq 0)$. Then for any $R, \delta>0$, there exists $\varepsilon=\varepsilon(n, k, R, \delta)$ such that if

$$
\sup _{x} \frac{1}{\operatorname{vol}(B(x, R))} \int_{B(x, R)}\left((n-1)-\mathrm{Ric}_{-}\right)_{+} d V<\varepsilon(n, k, R, \delta),
$$

then $(M, g)$ is compact, with $\operatorname{diam}(M)<\pi+\delta$.

Finally, we show that under similar conditions, one can also restrict the topology of $M$ as in Myers' Theorem.

Theorem 1.3. Let $(M, g)$ be a complete Riemannian manifold with Ric $\geq$ $(n-1) k(k \leq 0)$. Then for any $R>0$, there exists $\tilde{\varepsilon}=\tilde{\varepsilon}(n, k, R)$ such that if

$$
\sup _{x} \frac{1}{\operatorname{vol}(B(x, R))} \int_{B(x, R)}\left((n-1)-\mathrm{Ric}_{-}\right)_{+} d V<\tilde{\varepsilon}(n, k, R),
$$

then the universal cover of $M$ is compact, and hence $\pi_{1}(M)$ is finite.

We note that in the aforementioned papers, all theorems on the finiteness of $\pi_{1}(M)$ are proved in the class of Riemannian manifolds with Ric $\geq$ $(n-1) k$, diam $<D$, and vol $>v$. By a theorem of Anderson [1], this implies that the number of isomorphism classes possible for $\pi_{1}(M)$ is finite. However, in the above we merely require that our curvature quantity is small 
in an averaged sense, rather than assuming a strict lower volume bound on $(M, g)$. Hence there are an infinite number of possible isomorphism classes for $\pi_{1}(M)$. For instance, $S^{3} / \mathbb{Z}_{q}$ with constant sectional curvature 1 metric will always satisfy the hypotheses of Theorem 1.3. On the other hand, in the noncollapsing case one can also extend a result of [14] to achieve finiteness of $\pi_{1}(M)$ when no pointwise curvature bounds are assumed at all (Theorem 4.2 below).

As with [15], [14, Theorem 11.1], the proofs of Theorems 1.1-1.3 are geometric, being for the most part faithful to Myers' method in [10]. This is in contrast with [6], which depends on more analytic techniques. For recent probabilistic arguments, see [2] for an entirely different proof of the original theorem of Myers, and also [9] for results related to [14].

Acknowledgement. The author would like to thank Peter Petersen for very helpful advice and encouragement.

\section{Compact manifolds of nonnegative Ricci curvature.}

Let $A_{1}, A_{2}, W$ be open subsets of $M$ such that $A_{1}, A_{2} \subset W$, and all minimal geodesics $\gamma_{x, y}$ from $x \in A_{1}$ to $y \in A_{2}$ lie in $W . f$ will be any nonnegative integrable function on $M$.

In order to convert integral curvature bounds on $M$ into integral bounds along geodesics we will use the following estimate of Cheeger and Colding ([4, Theorem 2.11]):

$$
\begin{aligned}
& \int_{A_{1} \times A_{2}} \int_{\gamma_{x, y}} f(\gamma(s)) d s d V_{A_{1} \times A_{2}} \\
& \quad \leq C(n, k, R)\left(\operatorname{diam}\left(A_{2}\right) \operatorname{vol}\left(A_{1}\right)+\operatorname{diam}\left(A_{1}\right) \operatorname{vol}\left(A_{2}\right)\right) \int_{W} f d V .
\end{aligned}
$$

Where for $k \leq 0$,

$$
\begin{gathered}
C(n, k, R)=\frac{\operatorname{area}\left(\partial B_{k}(x, R)\right)}{\operatorname{area}\left(\partial B_{k}\left(x, \frac{R}{2}\right)\right)}, \\
R \geq \sup \left\{d(x, y) \mid(x, y) \in\left(A_{1} \times A_{2}\right)\right\},
\end{gathered}
$$

and $B_{k}(x, r)$ denotes the ball of radius $r$ in the simply-connected space of constant sectional curvature $k$. We will assume henceforth in this section that Ric $\geq 0$ on $M$, and thus $C(n, k, R)=C(n)$. 
$A_{1}$ and $A_{2}$ will be metric balls of small radius, $W=M$, and $f=((n-$ 1) - Ric $\left._{-}\right)_{+}$. We assume that all geodesics are parameterized by arclength and, by possibly removing a set of measure 0 in $A_{1} \times A_{2}$, that there is a unique minimal geodesic from $x$ to $y$ for all $(x, y) \in\left(A_{1}, A_{2}\right)$.

Proof of Theorem 1.1. Let $p, q \in M$ be such that $d(p, q)=\operatorname{diam}(M)=D$, $r>0, A_{1}=B(p, r), A_{2}=B(q, r)$.

Then (2.1) gives

$$
\begin{aligned}
\int_{A_{1} \times A_{2}} \int_{\gamma_{x, y}} & \left((n-1)-\mathrm{Ric}_{-}\right)_{+} d s d V_{A_{1} \times A_{2}} \\
\leq & 2 r C(n)\left(\operatorname{vol}\left(A_{1}\right)+\operatorname{vol}\left(A_{2}\right)\right) \int_{M}\left((n-1)-\mathrm{Ric}_{-}\right)_{+} d V
\end{aligned}
$$

which implies

$$
\begin{aligned}
\inf _{(x, y) \in A_{1} \times A_{2}} & \int_{\gamma_{x, y}}\left((n-1)-\mathrm{Ric}_{-}\right)_{+} d s \\
& \leq 2 r C(n)\left(\frac{1}{\operatorname{vol}\left(A_{1}\right)}+\frac{1}{\operatorname{vol}\left(A_{2}\right)}\right) \int_{M}\left((n-1)-\mathrm{Ric}_{-}\right)_{+} d V \\
& \leq 4 r C(n) \frac{D^{n}}{r^{n}} \frac{1}{\operatorname{vol}(M)} \int_{M}\left((n-1)-\mathrm{Ric}_{-}\right)_{+} d V
\end{aligned}
$$

where the final inequality follows from relative volume comparison. We can then find a minimizing unit-speed geodesic $\gamma$ from $x \in \bar{A}_{1}$ to $y \in \bar{A}_{2}$ which realizes this infimum, and will show that for $L=d(x, y)$ much larger than $\pi, \gamma$ cannot be minimizing if the right hand side of (2.5) is small enough.

Let $E_{1}(t), \ldots, E_{n}(t)=\gamma^{\prime}(t)$ be parallel, pointwise orthonormal vector fields along $\gamma, Y_{i}=\sin \left(\frac{\pi t}{L}\right) E_{i}(t), i=1, \ldots, n-1$. Then denoting by $L_{i}(s)$ the length functional of a fixed-endpoint variation of curves through $\gamma$ with variational vector field $Y_{i}$, we have by the second variation formula for arclength

$$
\begin{aligned}
\left.\sum_{i=1}^{n-1} \frac{d^{2} L_{i}}{d s^{2}}\right|_{s=0} & =\sum_{i=1}^{n-1} \int_{0}^{L} g\left(\nabla_{\gamma^{\prime}} Y_{i}, \nabla_{\gamma^{\prime}} Y_{i}\right)-R\left(\gamma^{\prime}, Y_{i}, \gamma^{\prime}, Y_{i}\right) d s \\
& =\int_{0}^{L}(n-1) \frac{\pi^{2}}{L^{2}} \cos ^{2}\left(\frac{\pi t}{L}\right)-\sin ^{2}\left(\frac{\pi t}{L}\right) \operatorname{Ric}\left(\gamma^{\prime}, \gamma^{\prime}\right) d s \\
& =(n-1) \int_{0}^{L} \frac{\pi^{2}}{L^{2}} \cos ^{2}\left(\frac{\pi t}{L}\right)-\sin ^{2}\left(\frac{\pi t}{L}\right) d s
\end{aligned}
$$




$$
\begin{aligned}
& +\int_{0}^{L} \sin ^{2}\left(\frac{\pi t}{L}\right)\left((n-1)-\operatorname{Ric}\left(\gamma^{\prime}, \gamma^{\prime}\right)\right) d s \\
= & -\frac{(n-1) L}{2}\left(1-\frac{\pi^{2}}{L^{2}}\right) \\
& +\int_{0}^{L} \sin ^{2}\left(\frac{\pi t}{L}\right)\left((n-1)-\operatorname{Ric}\left(\gamma^{\prime}, \gamma^{\prime}\right)\right) d s .
\end{aligned}
$$

And if the above quantity is negative, then there is a fixed endpoint variation $\gamma_{s}(t)$, satisfying

$$
\frac{d^{2}}{d s^{2}} \text { length }\left.\left(\gamma_{s}\right)\right|_{s=0}<0,
$$

and thus $\gamma=\gamma_{0}$ cannot minimize arclength.

But then,

$$
\begin{aligned}
\int_{0}^{L} \sin ^{2}\left(\frac{\pi t}{L}\right)((n-1) & \left.-\operatorname{Ric}\left(\gamma^{\prime}, \gamma^{\prime}\right)\right) d s \\
& \leq \int_{0}^{L} \sin ^{2}\left(\frac{\pi t}{L}\right)\left((n-1)-\mathrm{Ric}_{-}\right)_{+} d s \\
& \leq \int_{0}^{L}\left((n-1)-\mathrm{Ric}_{-}\right)_{+} d s \\
& \leq 4 r C(n) \frac{D^{n}}{r^{n}} \frac{1}{\operatorname{vol}(M)} \int_{M}\left((n-1)-\mathrm{Ric}_{-}\right)_{+} d V
\end{aligned}
$$

So suppose that we want to assure $D=\operatorname{diam}(M)<\pi+\delta$. Then letting $r=\frac{D}{N}$, choose $N=N(\delta)$ such that

$$
\frac{1}{1-\frac{2}{N}}<\frac{\pi+\delta}{\pi+\frac{\delta}{2}}
$$

By the triangle inequality,

$$
L=d(x, y) \geq d(p, q)-2 r=D\left(1-\frac{2}{N}\right) .
$$

So then showing that $L$ must be less than $\pi+\frac{\delta}{2}$ will finish the proof. By (2.6), $\gamma$ cannot be minimal if

$$
4 r C(n) \frac{D^{n}}{r^{n}} \frac{1}{\operatorname{vol}(M)} \int_{M}\left((n-1)-\mathrm{Ric}_{-}\right)_{+} d V<\frac{(n-1) L}{2}\left(1-\frac{\pi^{2}}{L^{2}}\right) .
$$


This, together with (2.10) implies that $\gamma$ cannot be minimizing if

$$
4 C(n) \frac{1}{1-\frac{2}{N}} \frac{N^{n-1}}{\operatorname{vol}(M)} \int_{M}\left((n-1)-\mathrm{Ric}_{-}\right)_{+} d V<\frac{(n-1)}{2}\left(1-\frac{\pi^{2}}{L^{2}}\right) .
$$

So then choosing

$$
\varepsilon=\frac{(n-1)\left(1-\frac{2}{N}\right)}{8 C(n) N^{n-1}}\left(1-\frac{\pi^{2}}{\left(\pi+\frac{\delta}{2}\right)^{2}}\right),
$$

we have that if

$$
\frac{1}{\operatorname{vol}(M)} \int_{M}\left((n-1)-\mathrm{Ric}_{-}\right)_{+} d V<\varepsilon
$$

then

$$
D=\operatorname{diam}(M) \leq \frac{L}{1-\frac{2}{N}}<\pi+\delta
$$

\section{Complete Manifolds of Ricci Curvature Bounded Below.}

We first assume that $R>\pi$. Then we will in fact show the following:

Theorem 3.1. Let $(M, g)$ be a complete Riemannian manifold with Ric $\geq$ $(n-1) k(k \leq 0)$. Then for any fixed $R>\pi$, there exists $\varepsilon=\varepsilon(n, k, R, \delta)$ such that if

$$
\frac{1}{\operatorname{vol}(B(p, R))} \int_{B(p, R)}\left((n-1)-\mathrm{Ric}_{-}\right)_{+} d V<\varepsilon(n, k, R, \delta),
$$

for some $B(p, R) \subset M$ then $M=B(p, R) \subset B(p, \pi+\delta)$.

Proof. Again we will use estimate (2.1). Fix $p \in M, W=B(p, R)$. Then $q$ will be any point in $W$ such that $\pi+4 r<d(p, q)<R-3 r$, where $0<r<\frac{1}{8}(R-\pi)$ is to be determined, and $A_{1}=B(p, r), A_{2}=B(q, r)$. From the triangle inequality, all minimal geodesics from $x \in \bar{A}_{1}$ to $y \in \bar{A}_{2}$ lie in $W$. 
As in the proof of Theorem 1.1, there is a geodesic $\gamma$ of length $L$ from $x \in \bar{A}_{1}$ to $y \in \bar{A}_{2}$ with

$$
\begin{aligned}
& \int_{\gamma}\left((n-1)-\mathrm{Ric}_{-}\right)_{+} d s \\
& \leq 2 r C(n, k, R)\left(\frac{1}{\operatorname{vol}(B(p, r))}\right. \\
& \left.\quad+\frac{1}{\operatorname{vol}(B(q, r))}\right) \int_{B(p, R)}\left((n-1)-\mathrm{Ric}_{-}\right)_{+} d V \\
& \leq 2 r C(n, k, R)\left(\frac{v_{k}(R)}{v_{k}(r)} \frac{1}{\operatorname{vol}(B(p, R))}\right. \\
& \left.\quad+\frac{v_{k}(2 R)}{v_{k}(r)} \frac{1}{\operatorname{vol}(B(q, 2 R))}\right) \int_{B(p, R)}\left((n-1)-\mathrm{Ric}_{-}\right)_{+} d V \\
& \leq 2 r C(n, k, R)\left(\frac{v_{k}(R)}{v_{k}(r)}\right. \\
& \left.\quad+\frac{v_{k}(2 R)}{v_{k}(r)}\right) \frac{1}{\operatorname{vol}(B(p, R))} \int_{B(p, R)}\left((n-1)-\mathrm{Ric}_{-}\right)_{+} d V
\end{aligned}
$$

Now suppose we want to show that $B(p, R) \subset B(p, \pi+\delta)$, where $\delta<$ $\frac{1}{2}(R-\pi)$. Then fixing $r=\frac{1}{4} \delta$, and proceeding as in Theorem 1.1, we have that (3.2) implies that $\gamma$ cannot be minimizing if

$$
\begin{aligned}
\frac{1}{2} \delta C(n, k, R) \frac{v_{k}(R)+v_{k}(2 R)}{v_{k}\left(\frac{1}{4} \delta\right)} \frac{1}{\operatorname{vol}(B(p, R))} \int_{B(p, R)} & \left((n-1)-\mathrm{Ric}_{-}\right)_{+} d V \\
\leq & \frac{(n-1) L}{2}\left(1-\frac{\pi^{2}}{L^{2}}\right) .
\end{aligned}
$$

So setting

$$
\varepsilon=\frac{2}{\delta C(n, k, R)} \frac{v_{k}\left(\frac{1}{4} \delta\right)}{v_{k}(R)+v_{k}(2 R)} \frac{(n-1)\left(\pi+\frac{1}{2} \delta\right)}{2}\left(1-\frac{\pi^{2}}{\left(\pi+\frac{1}{2} \delta\right)^{2}}\right)
$$

we have that if

$$
\frac{1}{\operatorname{vol}(B(p, R))} \int_{B(p, R)}\left((n-1)-\mathrm{Ric}_{-}\right)_{+} d V<\varepsilon,
$$

then the minimizing geodesic $\gamma$ from $B(p, r)$ to $B(q, r)$ must have length $L \leq \pi+\frac{1}{2} \delta$. So then $D=d(p, q) \leq \pi+\delta$. Hence for all points $q \in B(p, R-3 r)$ 
we have $d(p, q) \leq \pi+\delta$. And since $\pi+\delta<R-3 r$, this implies that no geodesic emanating from $p$ of length greater than $\pi+\delta$ can be length minimizing. Therefore $M=B(p, R)=B(p, R-3 r) \subset B(p, \pi+\delta)$.

By the triangle inequality, one also has:

Corollary 3.2. Let $(M, g)$ be a complete Riemannian manifold with Ric $\geq$ $(n-1) k(k \leq 0)$. Then for any fixed $R>\pi$, there exists $\varepsilon=\varepsilon(n, k, R, \delta)$ such that if

$$
\frac{1}{\operatorname{vol}(B(p, R))} \int_{B(p, R)}\left((n-1)-\mathrm{Ric}_{-}\right)_{+} d V<\varepsilon(n, k, R, \delta)
$$

for some $B(p, R) \subset M$, then $\operatorname{diam}(M)<2(\pi+\delta)$.

Theorem 3.1 shows that Theorem 1.2 holds for $R>\pi$. We are then left with showing the result for $R \leq \pi$.

Proof of Theorem 1.2.

Let $R^{\prime}>\pi$ be fixed. Then for any $R \leq \pi$, we have as is standard ([8]) that there is $N=N\left(k, R, R^{\prime}\right)$ such that any $R^{\prime}$-ball in $M$ can be covered by $N$ or fewer $R$-balls, $B\left(x_{i}, R\right)$. So then,

$$
\begin{aligned}
& \frac{1}{\operatorname{vol}\left(B\left(z, R^{\prime}\right)\right)} \int_{B\left(z, R^{\prime}\right)}\left((n-1)-\mathrm{Ric}_{-}\right)_{+} d V \\
& \leq N\left(k, R, R^{\prime}\right) \frac{1}{\operatorname{vol}\left(B\left(z, R^{\prime}\right)\right)} \sup _{x_{i}} \int_{B\left(x_{i}, R\right)}\left((n-1)-\mathrm{Ric}_{-}\right)_{+} d V \\
& \leq N\left(k, R, R^{\prime}\right) \frac{v_{k}\left(R+R^{\prime}\right)}{v_{k}\left(R^{\prime}\right)} \frac{1}{\operatorname{vol}\left(B\left(z, R+R^{\prime}\right)\right)} \\
& \quad \cdot \sup _{x_{i}} \int_{B\left(x_{i}, R\right)}\left((n-1)-\mathrm{Ric}_{-}\right)_{+} d V \\
& \leq N\left(k, R, R^{\prime}\right) \frac{v_{k}\left(R+R^{\prime}\right)}{v_{k}\left(R^{\prime}\right)} \\
& \cdot \sup _{x_{i}} \frac{1}{\operatorname{vol}\left(B\left(x_{i}, R\right)\right)} \int_{B\left(x_{i}, R\right)}\left((n-1)-\mathrm{Ric}_{-}\right)_{+} d V .
\end{aligned}
$$

And thus we have

$$
\begin{aligned}
& \text { (3.8) } \sup _{x} \frac{1}{\operatorname{vol}\left(B\left(x, R^{\prime}\right)\right)} \int_{B\left(x, R^{\prime}\right)}\left((n-1)-\mathrm{Ric}_{-}\right)_{+} d V \\
& \leq N\left(k, R, R^{\prime}\right) \frac{v_{k}\left(R+R^{\prime}\right)}{v_{k}\left(R^{\prime}\right)} \sup _{x} \frac{1}{\operatorname{vol}(B(x, R))} \int_{B(x, R)}\left((n-1)-\mathrm{Ric}_{-}\right)_{+} d V .
\end{aligned}
$$




\section{Integral curvature bounds and the fundamental group.}

We now show that choosing

$$
\sup _{x} \frac{1}{\operatorname{vol}(B(x, R))} \int_{B(x, R)}\left((n-1)-\mathrm{Ric}_{-}\right)_{+} d V
$$

small enough will imply compactness of the universal cover of $M$. As in the proof of Theorem 1.2, we will actually show something slightly more general than this. Theorem 1.3 will then follow from (3.8).

Theorem 4.1. Let $(M, g)$ be a complete Riemannian manifold with Ric $\geq$ $(n-1) k(k \leq 0)$. Then for any $R>2 \pi$, there exists $\tilde{\varepsilon}=\tilde{\varepsilon}(n, k, R)$ such that if

$$
\frac{1}{\operatorname{vol}(B(x, R))} \int_{B(x, R)}\left((n-1)-\mathrm{Ric}_{-}\right)+d V<\tilde{\varepsilon}(n, k, R),
$$

for some $B(x, R) \subset M$ then the universal cover of $M$ is compact, and hence $\pi_{1}(M)$ is finite.

Proof. Let $R>2 \pi$. By Corollary 3.2 we can assume that $\operatorname{diam}(M)<R$. So it is then sufficient to show that

$$
\frac{1}{\operatorname{vol}(B(\tilde{x}, R))} \int_{B(\tilde{x}, R)}\left((n-1)-\mathrm{Ric}_{-}\right)_{+} d V \rightarrow 0
$$

for $\tilde{x}$ in $\tilde{M}$ as

$$
\frac{1}{\operatorname{vol}(M)} \int_{M}\left((n-1)-\mathrm{Ric}_{-}\right)_{+} d V \rightarrow 0
$$

Let $N$ denote the minimal number of fundamental domains in $\tilde{M}$ necessary to cover $B(\tilde{x}, R)$. Then we have that

$$
\begin{aligned}
\frac{1}{\operatorname{vol}(B(\tilde{x}, R))} \int_{B(\tilde{x}, R)} & \left((n-1)-\mathrm{Ric}_{-}\right)_{+} d V \\
\leq & \frac{N}{\operatorname{vol}(B(\tilde{x}, R))} \int_{M}\left((n-1)-\mathrm{Ric}_{-}\right)_{+} d V \\
\leq & \frac{v_{k}(3 R)}{v_{k}(R)} \frac{N}{\operatorname{vol}(B(\tilde{x}, 3 R))} \int_{M}\left((n-1)-\mathrm{Ric}_{-}\right)_{+} d V \\
& \leq \frac{v_{k}(3 R)}{v_{k}(R)} \frac{N}{N \operatorname{vol}(M)} \int_{M}\left((n-1)-\mathrm{Ric}_{-}\right)_{+} d V \\
& =\frac{v_{k}(3 R)}{v_{k}(R)} \frac{1}{\operatorname{vol}(M)} \int_{M}\left((n-1)-\mathrm{Ric}_{-}\right)_{+} d V
\end{aligned}
$$


and the result follows.

It is worth pointing out that given the results in [7], [12], and [14] it is very possible that Theorems 1.2-1.3 are true with pointwise Ricci-curvature bound replaced by smallness in the $L^{p}$ norm of $\left(-\mathrm{Ric}_{-}\right)_{+}, p>\frac{n}{2}$. However (2.1) does not readily generalize to this case. We do have at least one obvious extension of a result in [14] to the case of $L^{p}$ curvature bounds, which states that given $(M, g)$ with upper diameter bound, lower volume bound, and lower sectional curvature bound, smallness of the $L^{1}$ norm of the Ricci curvature below a positive constant implies finite fundamental group. This is a consequence of Theorem 1.3 above, which does not require a sectional curvature bound, but it can also be slightly generalized in a different direction. Namely, in [14] the lower sectional curvature bound is used to control the 1-systole by Cheeger's method ([3]), which has been extended to $L^{p}$ curvature bounds in [11]. Therefore combining the proof of [14, Theorem 5] with [11] one has:

Theorem 4.2. Suppose $(M, g)$ satisfies $\operatorname{diam}(M)<D, \operatorname{vol}(M)>v$. Let $\Lambda \in \mathbb{R}, \lambda>0, p>n-1$. Then there is $\varepsilon_{1}=\varepsilon_{1}(n, p, v, D, \Lambda), \varepsilon_{2}=$ $\varepsilon_{2}(n, p, v, D, \lambda, \Lambda)$ such that if

$$
\int_{M}\left(\Lambda-\mathrm{sec}_{-}\right)_{+}^{p} d V<\varepsilon_{1}
$$

and

$$
\int_{M}\left(\lambda-\mathrm{Ric}_{-}\right)_{+} d V<\varepsilon_{2},
$$

then $M$ has finite fundamental group. Here, $\mathrm{sec}_{-}(x)$ denotes the infimum of sectional curvatures of 2-planes at $x$.

Proof. We follow the method of [14].

Let $A$ and $B$ be constants such that the Sobolev inequality

$$
\|f\|_{2 q /(q-1)}^{2} \leq A\|\nabla f\|_{2}^{2}+B\|f\|_{2}^{2}
$$

holds, where $\frac{n}{2}<q<p$. By the proof of [14, Theorem 10.1], if

$$
\int_{M}\left(\lambda-\mathrm{Ric}_{-}\right)_{+}^{q} d V<\min \left(A^{-1}, \lambda B^{-1}\right),
$$


then the first homology group $H^{1}(\bar{M}, \mathbb{R})$ vanishes for every finite cover $\bar{M}$ of $M$. If we take $B=v^{-\frac{1}{q}}$, then by [7] $A$ can be taken to be $A(q, v, D)$, under the assumption that

$$
\int_{M}\left(\lambda-\mathrm{Ric}_{-}\right)_{+}^{q} d V<\varepsilon_{3}(n, q, v, D)
$$

Given that $H^{1}(\bar{M}, \mathbb{R})=0$ for all $\bar{M}$, it is shown in [6] that if $\pi_{1}(M)$ is of polynomial growth, then $\pi_{1}(M)$ is in fact finite. By [14, Theorem 6], we have that this is true if

$$
\int_{M}\left(\lambda-\mathrm{Ric}_{-}\right)_{+}^{q} d V<\varepsilon_{4}(n, q, v, D, s)
$$

where $s$ is a lower bound for $\operatorname{sys}_{1}(M)$. To bound $\operatorname{sys}_{1}(M)$ from below, we use [11, Theorem 1.2], which shows that there is $\varepsilon_{1}(n, p, v, D, \Lambda)$ such that if

$$
\int_{M}\left(\Lambda-\mathrm{sec}_{-}\right)_{+}^{p} d V<\varepsilon_{1}
$$

then $\operatorname{sys}_{1}(M) \geq s(n, p, v, D, \Lambda)$. So then assuming (4.12) holds, we can choose

$$
\varepsilon_{5}=\min \left(A^{-1}, \lambda B^{-1}, \varepsilon_{3}, \varepsilon_{4}\right)=\varepsilon_{5}(n, p, q, v, D, \lambda, \Lambda) .
$$

Such that if

$$
\int_{M}\left(\Lambda-\sec _{-}\right)_{+}^{p} d V<\varepsilon_{1}
$$

and

$$
\int_{M}\left(\lambda-\mathrm{Ric}_{-}\right)_{+}^{q} d V<\varepsilon_{5}
$$

then $M$ has finite fundamental group. But then we also have 


$$
\begin{aligned}
& \int_{M}\left(\lambda-\mathrm{Ric}_{-}\right)_{+}^{q} d V \\
= & \int_{\left\{\mathrm{Ric}_{-}>\Lambda\right\}}\left(\lambda-\mathrm{Ric}_{-}\right)_{+}^{q} d V+\int_{\left\{\mathrm{Ric}_{-} \leq \Lambda\right\}}\left(\lambda-\mathrm{Ric}_{-}\right)_{+}^{q} d V \\
\leq & (\lambda-\Lambda)^{q-1} \int_{\left\{\mathrm{Ric}_{-}>\Lambda\right\}}\left(\lambda-\mathrm{Ric}_{-}\right)_{+} d V+2^{q}(\lambda-\Lambda)^{q} \operatorname{vol}\left(\left\{\mathrm{Ric}_{-} \leq \Lambda\right\}\right) \\
& +2^{q} \int_{\left\{\mathrm{Ric}_{-} \leq \Lambda\right\}}\left(\Lambda-\mathrm{Ric}_{-}\right)_{+}^{q} d V \\
\leq & (\lambda-\Lambda)^{q-1} \int_{\left\{\mathrm{Ric}_{-}>\Lambda\right\}}\left(\lambda-\mathrm{Ric}_{-}\right)_{+} d V+2^{q}(\lambda-\Lambda)^{q} \operatorname{vol}\left(\left\{\mathrm{Ric}_{-} \leq \Lambda\right\}\right) \\
& +2^{q} \operatorname{vol}\left(\left\{\mathrm{Ric}_{-} \leq \Lambda\right\}\right)^{\frac{p-q}{p}}\left(\int_{M}\left(\Lambda-\mathrm{Ric}_{-}\right)_{+}^{p} d V\right)^{\frac{q}{p}} \\
\leq & (\lambda-\Lambda)^{q-1}\left(1+2^{q}\right) \int_{M}\left(\lambda-\mathrm{Ric}_{-}\right)_{+} d V \\
& +2^{q}(\lambda-\Lambda)^{\frac{q-p}{p}}\left(\int_{M}\left(\lambda-\mathrm{Ric}_{-}\right)_{+} d V\right)^{\frac{p-q}{p}}
\end{aligned}
$$

where in the final inequality we have assumed that $\varepsilon_{1}$ was chosen to be less than 1 . So then we fix a $q>\frac{n}{2}$, and choose $\varepsilon_{2}=\varepsilon_{2}(n, p, v, D, \lambda, \Lambda)$ such that for

$$
\int_{M}\left(\lambda-\mathrm{Ric}_{-}\right)_{+} d V<\varepsilon_{2}
$$

the right hand side of the above equation is less than $\varepsilon_{5}$.

\section{References.}

[1] M.T. Anderson, Short geodesics and gravitational instantons, J. Differential Geom. 31 (1990), 265-275.

[2] D. Bakry and M. Ledoux, Sobolev inequalities and Myers's diameter theorem for an abstract Markov generator, Duke Math. J. 85 (1996), 253-270.

[3] J. Cheeger, Finiteness theorems for Riemannian manifolds, Amer. J. Math. 92 (1970), 61-74.

[4] J. Cheeger and T. H. Colding, Lower bounds on Ricci curvature and almost rigidity of warped products, Ann. Math. 144 (1996), 189-237. 
[5] K.D. Elworthy and S. Rosenberg, Compact manifolds with a little negative curvature, Bull. Amer. Math. Soc. 20 (1989), 41-44.

[6] K.D. Elworthy and S. Rosenberg, Manifolds with wells of negative curvature, appendix by D. Ruberman, Invent. Math. 103 (1991), 471-495.

[7] S. Gallot, Isoperimetric inequalities based on integral norms of Ricci curvature, Astérisque No. 157-158 (1988), 191-216.

[8] M. Gromov, J. Lafontaine, and P. Pansu, Structures métriques pour les variétés riemannienness, Paris: Cedic/Fernand, Nathan, 1981.

[9] X.-M. Li, On extensions of Myers' Theorem, Bull. London Math. Soc. 27 (1995), 392-396.

[10] S.B. Myers, Riemannian manifolds with positive mean curvature, Duke Math. J. 8 (1941), 401-404.

[11] P. Petersen, S. Shteingold, and G. Wei, Comparison geometry with integral curvature bounds, Geom. Funct. Anal. 7 (1997), 1011-1030.

[12] P. Petersen and G. Wei, Relative volume comparison with integral curvature bounds, Geom. Funct. Anal. 7 (1997), 1031-1045.

[13] P. Petersen and G. Wei, Analysis and geometry on manifolds with integral Ricci curvature bounds, preprint.

[14] S. Rosenberg and D. Yang, Bounds on the fundamental group of a manifold with almost nonnegative Ricci curvature, J. Math. Soc. Japan 46 (1994), 267286.

[15] J.-Y. Wu, Complete manifolds with a little negative curvature, Amer. J. Math. 113 (1991), 567-572.

UCLA

LOS ANGELES, CA 90095

E-mail address: csprouse@math.ucla.edu

Received March 4, 1998. 